Exploring simultaneous single and coincident gamma-ray measurements for $\mathrm{U} / \mathrm{Pu}$ assay in safeguards

T. F. Wang, S. M. Horne, R. A. Henderson, K. E. Roberts, D. K. Vogt

May 10, 2011

ANIMMA-The Second International Conference on Advancements in Nuclear Instrumentation, Measurement Methods and their Applications ICC, Ghnet, Belgium June 6, 2011 through June 9, 2011 
This document was prepared as an account of work sponsored by an agency of the United States government. Neither the United States government nor Lawrence Livermore National Security, LLC, nor any of their employees makes any warranty, expressed or implied, or assumes any legal liability or responsibility for the accuracy, completeness, or usefulness of any information, apparatus, product, or process disclosed, or represents that its use would not infringe privately owned rights. Reference herein to any specific commercial product, process, or service by trade name, trademark, manufacturer, or otherwise does not necessarily constitute or imply its endorsement, recommendation, or favoring by the United States government or Lawrence Livermore National Security, LLC. The views and opinions of authors expressed herein do not necessarily state or reflect those of the United States government or Lawrence Livermore National Security, LLC, and shall not be used for advertising or product endorsement purposes. 


\title{
Exploring simultaneous single and coincident gamma-ray measurements for U/Pu assay in safeguards
}

\author{
T.F. Wang ${ }^{1}$, S.M. Horne ${ }^{2}$, R.A. Henderson ${ }^{1}$, K.E. Roberts ${ }^{1}$, and D.K. Vogt ${ }^{1}$ \\ ${ }^{1}$ Lawrence Livermore National Laboratory, Livermore, California, USA \\ ${ }^{2}$ Research performed at Livermore National Laboratory, Livermore, California. Present address \\ University of Texas at Austin, Austin, Texas, USA
}

\begin{abstract}
:
Using a broad range of gamma-ray uranium standards and two plutonium samples of known isotopic content, list mode gamma ray information from two Compton suppressed and one planar HPGe detectors were analyzed according to the time information of the signals. Interferences from Cs-137 were introduced. In this study, we extended singles measurements by exploring the potential of simultaneously using both singles and coincidence data for U/Pu assay. The main goals of this exploratory study are: 1 ) whether one will be able to use coincidence information in addition to the complicated $100-\mathrm{keV}$ unfolding to obtain extra information of uranium and plutonium isotopic ratios, and 2) with higher energy interference gamma-rays from isotopes such as Cs-137, can the coincidence information help to provide the isotopic information.

\section{Introduction:}

Uranium and plutonium singles gamma-ray non-destructive assay has been proven to provide a measure of an accurate broad range uranium/plutonium isotopic content for safeguards. Codes such as MGA++ [1] and FRAM [2] have been developed and are widely used in the safeguards community using an HPGe gamma-ray detector system for this sole purpose.

The MGA++ code developed at Lawrence Livermore National Laboratory typically uses the low energy part $(<300-k e V)$ of the gamma-ray information from both uranium and plutonium that can be obtained with a planer detector with good energy resolution $(<600 \mathrm{eV} @ 120-\mathrm{keV})$, so that robust unfolding of complicated $100-\mathrm{keV}$ region for both uranium and plutonium gamma-ray spectra is possible. The unfolding mechanisms employed in the code for plutonium and uranium isotopic contents are shown in Figures $1(a)$ and $1(b)$, respectively. The dominant gamma-ray groups, for example, used in the unfolding of plutonium gamma-rays are listed in Table 1 . Since the uranium analysis relying on the daughter gamma-rays of U-238, either secular equilibrium between the U-238 parent and $\mathrm{Pa}-234$ daughters or known separation time is required. In general, for pure and homogeneous samples, MGA++ analysis provides good results.
\end{abstract}



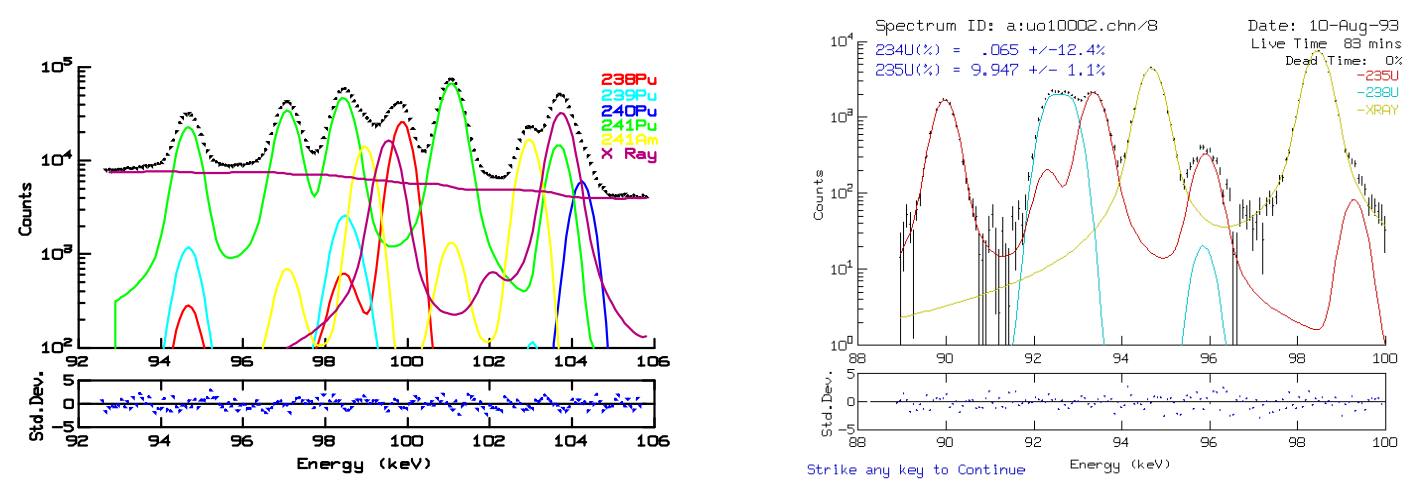

Figure 1 (a, left) MGA++ unfolding of the complicated 100-keV plutonium gamma-ray region, and (b, right) unfolding of 100-keV uranium gamm-ray region.

\begin{tabular}{|l|l|l|l|l|l|l|}
\hline Energy(keV) & \multicolumn{1}{|c|}{ Pu-239 } & Pu-240 & Pu-241 & Am-241 & X-ray & Np-237 \\
\hline 86.5 & 0 & 0 & 0 & 0 & 0 & 0.14 \\
\hline 94.66 & $4.20 \mathrm{E}-05$ & $6.80 \mathrm{E}-07$ & $2.98 \mathrm{E}-06$ & 0 & 0 & 0.0931 \\
\hline 95.858 & 0 & 0 & 0 & 0 & 0 & 0.029 \\
\hline 96.16 & $3.68 \mathrm{E}-07$ & 0 & 0 & 0 & 0 & 0 \\
\hline 97.071 & 0 & 0 & $3.80 \mathrm{E}-06$ & $1.20 \mathrm{E}-05$ & 0 & 0 \\
\hline 98.443 & $6.82 \mathrm{E}-05$ & $1.00 \mathrm{E}-06$ & $4.84 \mathrm{E}-06$ & 0 & 0 & 0.14 \\
\hline 98.79 & $1.22 \mathrm{E}-05$ & 0 & 0 & 0 & 0 & 0 \\
\hline 98.956 & 0 & 0 & 0 & $2.00 \mathrm{E}-04$ & 0 & 0 \\
\hline 99.533 & 0 & 0 & 0 & 0 & $4.14 \mathrm{E}-05$ & 0 \\
\hline 99.864 & 0 & 0 & 0 & 0 & 0 & 0 \\
\hline 101.063 & 0 & 0 & $6.12 \mathrm{E}-06$ & $1.94 \mathrm{E}-05$ & 0 & 0 \\
\hline 102.963 & 0 & 0 & 0 & $1.93 \mathrm{E}-04$ & 0 & 0 \\
\hline 103.035 & $2.12 \mathrm{E}-06$ & 0 & 0 & 0 & 0 & 0 \\
\hline 103.679 & 0 & 0 & $9.85 \mathrm{E}-07$ & 0 & 0 & 0 \\
\hline 103.742 & 0 & 0 & 0 & 0 & $6.64 \mathrm{E}-05$ & 0 \\
\hline 103.86 & 0 & 0 & 0 & 0 & 0 & 0.0083 \\
\hline 104.242 & 0 & $6.97 \mathrm{E}-05$ & 0 & 0 & 0 & 0 \\
\hline
\end{tabular}

Table 1 Gamma-ray branching ratios used in MGA++ for $100-k e V$ plutonium unfolding.

In this study, we extended singles measurements by exploring the potential of simultaneously using both singles and coincidence data for U/Pu assay. The main goals of this exploratory study are: 1 ) whether one will be able to use coincidence information in addition to the complicated 100-keV unfolding to obtain extra information of uranium and plutonium isotopic ratios, and 2) with higher energy interference gamma-rays from isotopes such as Cs-137, can the coincidence information help to provide the isotopic information.

Some dominant coincidence gamma-rays for uranium and plutonium are listed in Table 2 [3] as well as the energy of the excite state in the daughter nuclides. It is also important to note that because of the high $-Z$ internal conversion as well as multipolarity of the decay, the actually observable coincidence gamma-rays may not be the pair on the list. Most of the coincidence gamma-rays are low-energy and mostly from U-235 decay; the U-238 coincidences seldom observed because coincidences gamma-rays 
are in a pattern of high- energy (low-detection efficiency) - low energy (high internal conversion) behavior. This is one reason we are using single high-energy gamma-ray information of U-238 daughter decay (e.g., 1001-keV and 766-keV, etc) to obtain the U-238 information in the sample. Similar coincidence behaviors in the plutonium decay are seen from the list.

\begin{tabular}{|l|l|l|l|}
\hline Isotope & Energy(keV) & Energy(keV) & Excitation Energy(keV, daughter) \\
\hline $235 \mathrm{U}$ & 41.95 & 143.764 & 185.714 \\
\hline $235 \mathrm{U}$ & 42.952 & 163.358 & 205.309 \\
\hline $235 \mathrm{U}$ & 96.09 & 140.76 & 236.893 \\
\hline $235 \mathrm{U}$ & 41.95 & 194.94 & 236.893 \\
\hline $235 \mathrm{U}$ & 96.09 & 109.16 & 305.309 \\
\hline $235 \mathrm{U}$ & 185.714 & 202.111 & 387.827 \\
\hline $238 \mathrm{U}(234 \mathrm{Th})$ & 29.49 & 63.29 & $166.72+x$ \\
\hline $238 \mathrm{U}(234 \mathrm{~Pa})$ & 43.49 & 946 & 989.45 \\
\hline $238 \mathrm{U}(234 \mathrm{~Pa})$ & 43.49 & 1001 & 1044.53 \\
\hline $234 \mathrm{U}$ & 53.2 & 120.9 & 174.1 \\
\hline $239 \mathrm{Pu}$ & 56.828 & 46.204 & 103.032 \\
\hline $239 \mathrm{Pu}$ & 51.624 & 98.78 & 150.453 \\
\hline $240 \mathrm{Pu}$ & 45.24 & 104.234 & 149.476 \\
\hline $240 \mathrm{Pu}$ & 104.234 & 160.308 & 309.784 \\
\hline $241 \mathrm{Pu}$ & 71.6 & 121.2 & 204.19 \\
\hline $241 \mathrm{Pu}(237 \mathrm{U})$ & 59.537 & 208 & 267.54 \\
\hline $241 \mathrm{Pu}(237 \mathrm{U})$ & 59.537 & 221.8 & 281.35 \\
\hline
\end{tabular}

Table $2 \mathrm{~A}$ lists of coincidence gamma-rays for $\mathrm{U}$ and $\mathrm{Pu}$.

\section{Experimental setup:}

List mode data acquisition system MPA-3 from FAST ComTec [4] was used. The system has an internal clock that generates a 50nS accuracy time stamp and writes an event-by-event file to the computer hard disk. Coincidence and single data can then be sorted according to the signal arrival in time. Two HPGe detectors (e.g., GEM-30P4) from ORTEC with custom made bismuth germanate (BGO) suppression shields and a planar detector were used in this study (Figure 2). Both Compton-suppressed detectors sit on separate laser tables supported by gears with sub-mm geometry precision. The preamplifier signals from HPGe detectors were feed into the ORTEC 672 amplifies with shaping time of 6uS, unipolar signals were feed into the Quanta 7072 analogue-to-digital converters (ADCs) which have a fixed conversion time of 500nS. Anode signals from BGOs were combined and sent to an ORTEC 579 timing filter amplifier and generate gates via Lecroy 621 and ORTEC 416A and then feed into the 7002 ADCs for signal conversion rejection. We encountered spurious gamma-ray peaks issues when associating pile-up gates from amplifier with the ADCs, therefore, no pile-up rejection was employed. 


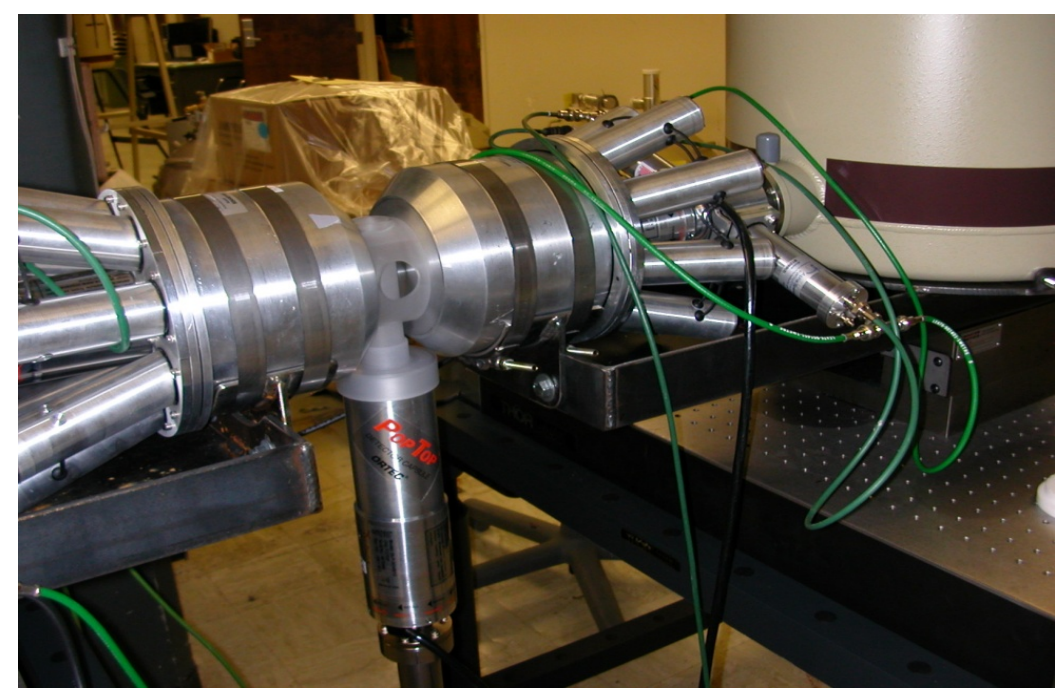

Fig 2. Two side-by-side Compton suppression detectors and a planar detector used in this study.

A set of twelve one-gram uranium gamma-ray standards (ranging from depleted uranium (DU) to highly enriched uranium (HEU)) from National Bureau of Standards was used in this study along with two plutonium sources: a sealed weapon grade plutonium gamma-ray standard from plutonium intercomparison exercise (PIDIE) [5] and a extremely pure Pu-239 ( > 99.94\%) sample. The data were collected in various time intervals ranging from one hour to twelve hours depending on the uranium enrichment of the standards. Three Cs-137 sources of activity about $0.5-, 2-, 5-$, and $10-u C i$ at counting time were used.

\section{Experimental results:}

We first studied the interference due to Cs-137 source in the single measurements. Figure 3 shows a plot of $5 \mathrm{uCi}$ of Cs-137 source with the $93 \% 1 \mathrm{~g}-\mathrm{HEU}(\sim 1.5 \mathrm{E}-6 \mathrm{uCi})$ standard (top), and only the $93 \%$ source (bottom). The plot already demonstrates possible problematic of performing a MGA++ unfolding using the 100-keV region. The Cs-137 backscatter also produces a bump just under the strong 186-keV gamma-ray from U-235 decay which can perturb analyses that use 186-keV gamma-ray information (e.g. enrichment meter, etc.). The results from MGA++ fits of three high enriched uranium standards and a depleted uranium standard are shown in Table 3 along with different strengths of Cs-137 sources. The results show that the uranium analysis in the MGA++ is quite robust up to few uCi of Cs-137 interferences. However, at the two extrema (weapon grade and depleted uranium) when the Cs-137 interferences are of the order of $5 \mathrm{uCi}$, interferences dominate. The MGA++ algorithms also show interesting departure among the two extremes with high Cs-137 interferences: For weapon grade uranium, significant lower in the enrichment value, and for depleted uranium, significant errors. For PIDIE plutonium (450mg, 25mCi, $>30$ year age) study, the MGA++ results are shown in Table 4. Higher (> 10uCi) Cs-137 activity is needed to degrade MGA++ results. 


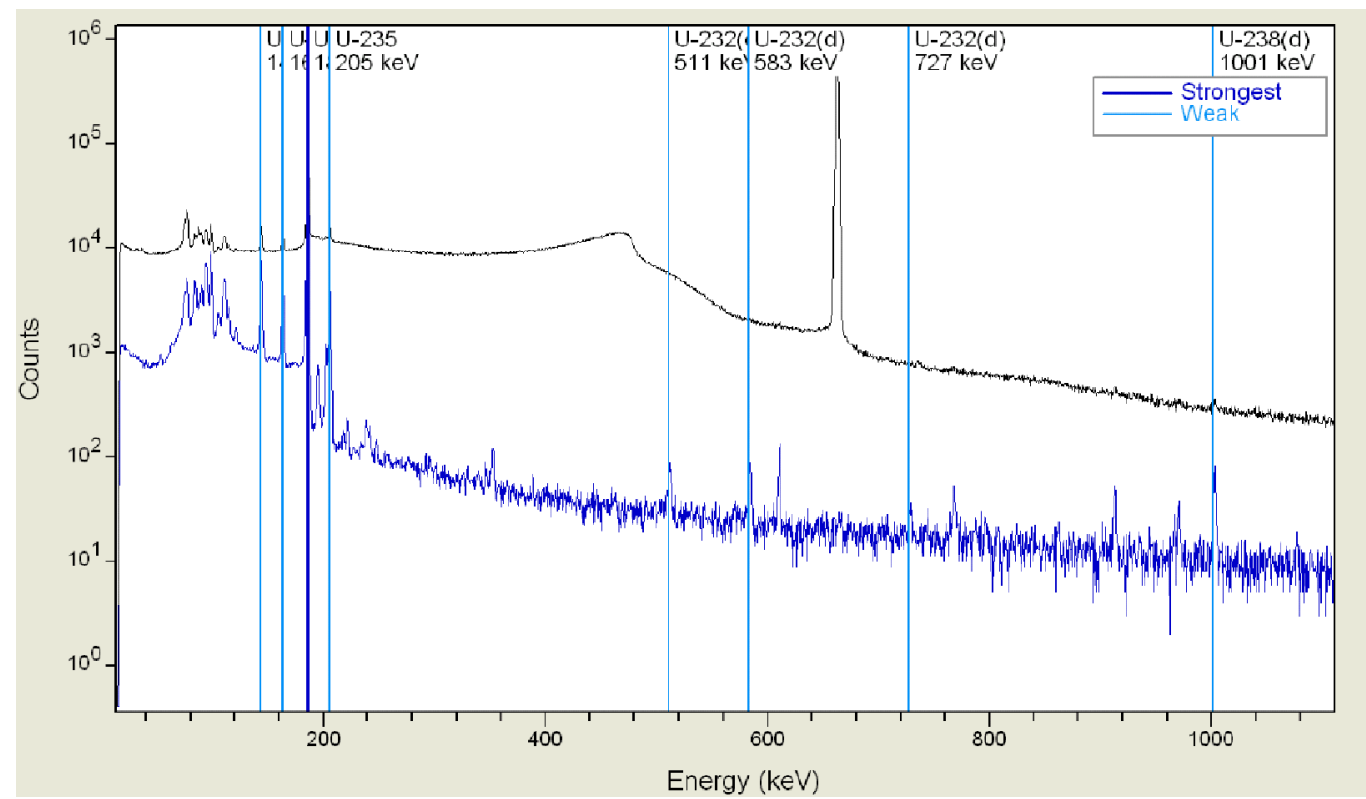

Figure 3 Gamma-rays from high-enriched uranium standards (bottom line); with 5uCi Cs-137 (top line).

\begin{tabular}{|l|l|l|l|l|l|}
\hline Standards & No Cs-137 & with 0.5uCi & with 2uCi & with 5uCi & with 10uCi \\
\hline U930 & $93.4+-2.3$ & $93.09+-2.4$ & $91.4+-1.7$ & $76.03+0.4$ & $65.46+-0.42$ \\
\hline U750 & $78.1+-0.1$ & $77.6+-0.1$ & $78.5+0.1$ & $79.1+-0.1$ & $79.3+-0.5$ \\
\hline U500 & $50.3+-0.05$ & $49.5+-0.07$ & $50.7+-0.1$ & $51.7+-0.1$ & $53.6+-0.2$ \\
\hline U005 & $0.57+-0.05$ & $0.55+-0.04$ & $0.49+-0.04$ & $0.41+-0.43$ & $0.64+-0.32$ \\
\hline
\end{tabular}

Table 3 Cs-137 interference study, the first column is MGA++ results with no Cs-137 source, the second column is the results with $0.5-\mathrm{uCi}$ of Cs-137 source, etc.

\begin{tabular}{|l|l|l|l|l|}
\hline PIDIE & NoCs-137 & with 5uCi & with 10uCi & with 25uCi \\
\hline Pu-239 & $93.8+-0.03$ & $93.7+-0.03$ & $93.9+-0.05$ & $94.3+-0.18$ \\
\hline Pu-240 & $6.01+-0.45$ & $6.02+-0.45$ & $5.95+-0.05$ & $5.56+-0.16$ \\
\hline Pu-eff & $6.07+-0.24$ & $6.06+-0.24$ & $6.01+-0.05$ & $5.63+-0.16$ \\
\hline
\end{tabular}

Table 4 Cs-137 study with a weapon grade plutonium inter-comparison exercise (PIDIE) source, the Pu239, Pu-240 and Pu-effective results from MGA++ analysis are shown.

We carried out coincidence measurements to hopefully compensate some of the deficiencies in using only the information from singles. Figure 4 (a) shows a coincidence matrix with a highly enriched uranium standard and 4 (b) is from a depleted uranium standard, using replay routine provided by the FAST ComTec. There are several features (apart from the true coincidences) in the coincidence matrix: pile-up, random, and backscattered. One interesting feature is in the backscatter behavior of the gamma-ray that forms an parallel to the off-diagonal line (upper left to lower right) in the $x-y$ coincidence matrix: the electron energy deposit in the detector while gating on the backscattered photo peak will summed up to the real energy of the true incidence gamma-ray. Because of high resolution of the HPGe detection system, one can distinguish the electron energy deposited in the detector by the broad width of the peak. 

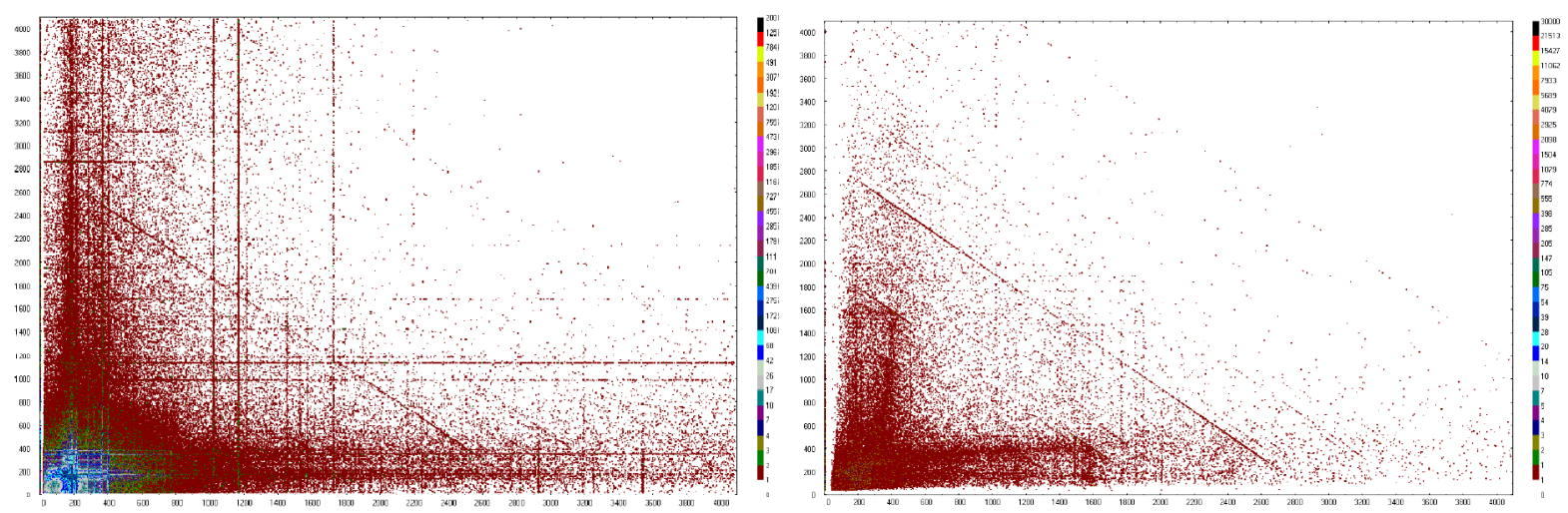

Figure 4 (a, left) High-enriched unranium coincidence matrix, (b,right) Depleted-uranium coincidence matrix.

A typical gated coincidence gamma-ray spectrum with a gate on the 186-KeV U-235 gamma-rays is shown in Figure 5. It is important to note that the 144-163-186-205-keV gammas are in the same intensity ratios as in the singles and, therefore, are random events. One of the immediate observed coincidence gamma rays with $186-\mathrm{keV}$ is the $202-\mathrm{keV}$. By gating on each of the gamma-rays that list in the Table 2 of U-235 gamma decay, it seems for the uranium study the most promising one is the 202$186-k e V$ pair with our system. It is possible that our system may have not optimized for some of the coincidence combinations. For U-238 coincidences even with depleted $U$ standard, we are unable to observe any of the coincidence pairs that were listed in Table 2 within 12-hours of collection time. We have therefore used 185-202-keV coincidence pair for U-235 determination and using 1001-keV single gamma from U-238 determination. For the pure Pu-239 study, we are able to observe several coincidence pairs. The most practical pair under our configuration is $129-203-k e V$ which is not on the list in Table 2. We are yet to complete the rest of the studies with other plutonium samples and standards.

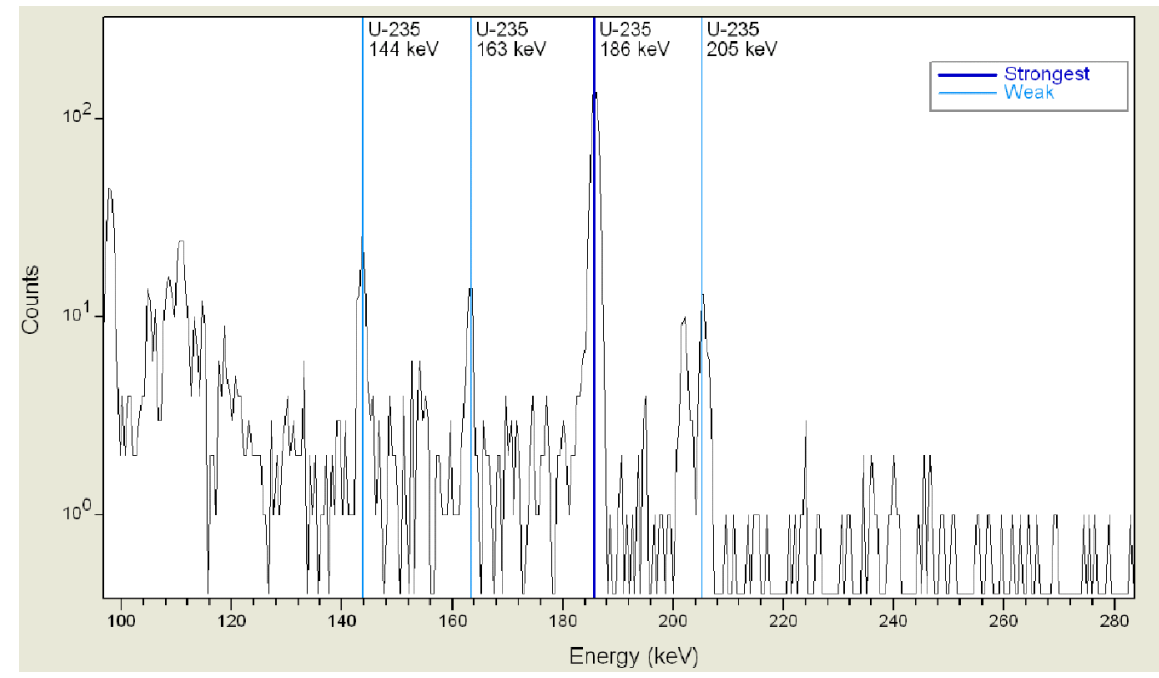

Figure 5 Gamma-rays in coincidence with 186-keV from U-235, the 202-keV gamma-ray shows noticeable enhancement; the 144-163-186-205 gammas are from random coincidence. 
To characterize the U-238 in a sample, we take single spectrum and analyze the peak from the 1001-keV photon of $\mathrm{Pa}-234 \mathrm{~m}$, with the assumption that the sample already reached parent-daughter equilibrium. By looking at a series of known standards, one is able to characterize a particular detector setup by creating an equation relating the isotopic ratio of uranium to the ratio of events characterizing U-235 and the events characterizing $\mathrm{U}-238$, the isotopic ratio of uranium can be obtained [6]. For our system, the uranium enrichment is approximately inversely proportional to $(1+0.22 *$ single count-rate [1001keV]/Coincidence count-rate [186-keV-202-keV pair]).

This 0.22 factor is a function of: 1) absolute gamma-ray decay branching ratio, 2) self absorption, and 3) detection efficiency of the selected single and coincidence gamma-rays. Moreover, it also contained the angular-correlation geometry coefficients of the coincidence pair.

We then applied this empirical determination for highly- enriched and depleted uranium standards with the introduction of Cs-137 interferences as in Table 3, the observed isotopic enrichment effects are shown in Table 5. It is clearly shown that for sources without interferences, the MGA++ results from singles provide higher precisions; however, for increasing Cs-137 interferences, the coincidence results still provide a solid picture of uranium enrichment.

\begin{tabular}{|l|l|l|l|l|l|}
\hline Standards & No Cs & with 0.5uCi & with 2uCi & with 5uCi & with 10uCi \\
\hline U930 & $91.2+-2.6$ & $91.0+-3.3$ & $90.9+-3.6$ & $91.4+-4.0$ & $90.3+-4.2$ \\
\hline U750 & $76.0+-3.2$ & $76.3+-3.7$ & $76.2+-3.8$ & $76.2+-3.8$ & $76.1+-4.0$ \\
\hline U500 & $52.3+-4.0$ & $53.7+-4.1$ & $52.6+-4.5$ & $53.5+-4.5$ & $53.0+-4.6$ \\
\hline U005 & $0.52+-0.1$ & $0.53+-0.1$ & $0.56+-0.1$ & $0.55+-0.1$ & $0.53+-0.1$ \\
\hline
\end{tabular}

Table 5 Coincidence results of the uranium enrichment with and without Cs-137 interferences as described in Table 3.

\section{Conclusions:}

Using information from both single and coincidence measurements simultaneously can provide additional knowledge of the sample. This approach can be used in, for example, facilities that perform special nuclear materials accountability with two detectors (e.g., MGA++ in two-detector mode, etc.). From this study we have learned that coincidence information can be used to strengthen uranium enrichment determination. However, to what extent this method can be extended (e.g., how to use single information properly account for both attenuation and self shielding as well as detector efficiencies to the coincidence information, etc.) and used for uranium samples with various interference gamma-rays still needs further investigation. Although not investigated here, this approach may also be applied to MOX (mixed oxide) fuel with various U/Pu weight fractions and different $\mathrm{U} / \mathrm{Pu}$ isotopic composition.

It is worth noting that: 1) Compton suppression provide almost no advantages in the uranium accountability studies because of the low energy characteristic behavior of the U-235 decay, although it can provide some improvement to remove Compton continuum from high-energy interfering gammarays, unfortunately, the Compton suppression will not be able to suppress the Compton effects from the 
source, 2) One of the two coincidence energy pair from U/Pu mostly is low in energy therefore the high$Z$ internal conversion reduces the coincidence efficiency dramatically, moreover, imposing coincidence conditions already presented a factor of $\sim 100$ (or more) reduction in the detection efficiency for almost every case in this study, and 3) Cumulative decay intensities for strong decay gamma-rays, in general, have been studied quite precisely for both uranium and plutonium, unfortunately, the gamma-ray decay branches for a given level are not quite precise, nuclear database revision is need to unfold properly the coincidence measurements. One should perform more precise studies to strengthen current nuclear database and also perform studies with careful selection of optimized gamma-ray detection systems to achieve an absolute determination (i.e., without perform empirical determination for a particular experimental setup) of the isotopic compositions.

\section{Acknowledgements:}

This work performed under the auspices of the U.S. Department of Energy by Lawrence Livermore National Laboratory under Contract DE-AC52-07NA27344. This work was funded by National Nuclear Security Agency's Office of Nonproliferation and Verification Research and Development Assessment, NA-22.

\section{References:}

[1] D. Clark, T.F. Wang, et al. "Uranium and plutonium isotopic analysis using MGA++," $39^{\text {th }}$ Annual Institute of Nuclear Materials Management Meeting, July, 1998.

[2] T. E. Sampson, T. A. Kelley, D. T. Vo, "Application guide to gamma ray isotopic analysis using the FRAM software," Los Alamos National Laboratory report LA-14018, June, 2003.

[3] R.B Firestone et al. "Table of Isotopes," John Wiley \& Sons, 1996, and reference therein.

[4] Fast Comtec : www.fastcomtec.com

[5] J. Moerel, and Bruno Chauvenet, "Intercomparison des measures de composition isotopique du plutonium par spectrometrie $x$ et gamma resulatats de l'acrion "PIDIE" rapport final," Commissariat a L'Energie Atomique, CEA-R-5582, 1991

[6] S. M. Horne, T.F. Wang," Uranium and plutonium isotope detection using coincidence methods " LLNL-POST-446391, 2010, and S. M. Horne, Master Thesis, Chapter 10 and 11, University of Texas at Austin, 2011 